\title{
Separability conditions from the Landau-Pollak uncertainty relation
}

\author{
Julio I. de Vicente ${ }^{1}$ and Jorge Sánchez-Ruiz ${ }^{1,2}$ \\ ${ }^{1}$ Departamento de Matemáticas, Universidad Carlos III de Madrid, \\ Avda. de la Universidad 30, 28911 Leganés, Madrid, Spain \\ ${ }^{2}$ Instituto Carlos I de Física Teórica y Computacional, \\ Universidad de Granada, 18071 Granada, Spain
}

\begin{abstract}
We obtain a collection of necessary (sufficient) conditions for a bipartite system of qubits to be separable (entangled), which are based on the Landau-Pollak formulation of the uncertainty principle. These conditions are tested, and compared with previously stated criteria, by applying them to states whose separability limits are already known. Our results are also extended to multipartite and higher-dimensional systems.
\end{abstract}

PACS numbers: 03.67.Mn, 03.65.Ud, 03.65.Ta 


\section{INTRODUCTION}

Consider the vector $|\psi\rangle$, pertaining to a finite-dimensional Hilbert space $H=H_{A} \otimes H_{B}$, that describes a pure state of two quantum systems $A$ and $B$. $|\psi\rangle$ is said to be a product state if there exists $|\phi\rangle_{A} \in H_{A}$ and $|\varphi\rangle_{B} \in H_{B}$ such that

$$
|\psi\rangle=|\phi\rangle_{A} \otimes|\varphi\rangle_{B}
$$

Separable states are mixtures of product states. In other words, the density operator $\rho$ acting on $H$ that characterizes the quantum state of $A$ and $B$ is called separable if it can be written as a convex combination of product vectors, that is,

$$
\rho=\sum_{i} p_{i}\left|\phi_{i}, \varphi_{i}\right\rangle\left\langle\phi_{i}, \varphi_{i}\right|=\sum_{i} p_{i} \rho_{i}^{A} \otimes \rho_{i}^{B}
$$

where $0 \leq p_{i} \leq 1, \sum_{i} p_{i}=1$, and $\left|\phi_{i}, \varphi_{i}\right\rangle=\left|\phi_{i}\right\rangle_{A} \otimes\left|\varphi_{i}\right\rangle_{B}$.

If $\rho$ cannot be written as in Eq. (2), then the state is said to be entangled. Entanglement is one of the most fascinating issues in quantum mechanics, not only from a theoretical point

of view [1], but also because of its applications in the context of quantum information theory, such as cryptography and teleportation [2]. Therefore, it is a very interesting question to ask whether a given state is entangled or not. Although no general answer is known, there exist a great variety of separability criteria, like the partial transpose criterion [3]. Bell's inequalities violation [4], and the construction of entanglement witnesses (EW's) [5]. The first of these criteria gives necessary and sufficient conditions when the dimension of $H$ is either $2 \times 2$ or $2 \times 3$, while otherwise it is just a necessary condition. The second criterion provides only a necessary condition. Finally, the third criterion is necessary and sufficient in the sense that, given an entangled state, there always exists an EW that detects it; however, it is not known how to construct all possible EW's, and this criterion turns out to be a necessary separability condition once a particular set of EW's has been chosen.

The relationship between entanglement and the uncertainty principle has been investigated in several recent works (see e.g. [6]). The key fact is that, when measuring a collection of nonlocal observables on a given state, the lower bound on the uncertainty of the outcomes is higher for separable states than for entangled states, because of the correlations inherent in the latter. Nonlocal operators possess, in general, entangled eigenstates, while separable states cannot be simultaneous eigenstates for the set of nonlocal operators. Using this idea, 
there have been achieved variance-based separability criteria [7] inspired by the HeisenbergRobertson formulation of the uncertainty principle [8], as well as entropy-based separability criteria [9, 10] derived from entropic uncertainty relations [11, 12, 13]. The necessary separability conditions obtained in this way have the advantage of being more easily implemented in experiments, since they are based on expectation values and probabilities for the outcomes of measurements. On the contrary, the partial transpose criterion demands complete knowledge of the density matrix, whose experimental determination requires considerable effort.

In this paper we derive new separability criteria based on a different mathematical formulation of the uncertainty principle, the so-called Landau-Pollak uncertainty relation, and we show that these conditions are better than those obtained using entropies in the examples proposed so far. The article is organized as follows. The Landau-Pollak uncertainty relation is briefly reviewed in Sec. II, where we state some properties that will be useful later on. In Sec. III, we derive new separability conditions for two-qubit systems. In Sec. IV, we investigate the accuracy of the resulting criteria using some well-known examples. In Sec. $\mathrm{V}$, the relationship between one of our separability conditions and a set of optimal EW's is pointed out. Section VI deals with the extension of our approach to more complex cases, i.e. bipartite systems of qudits and multipartite systems.

\section{THE LANDAU-POLLAK UNCERTAINTY RELATION}

Let $X$ denote a Hermitian operator representing some physical observable in a finitedimensional Hilbert space of dimension $D$, with a complete set of orthonormal eigenvectors $\left\{\left|x_{i}\right\rangle\right\}(i=1,2, \ldots, D)$ and $N$ distinct eigenvalues $(N \leq D)$. For $n=1,2, \ldots, N$, the probability $p_{n}(X)$ of finding the state $\rho$ in the $n$th eigenspace of $X$ (i.e., the probability of obtaining the $n$th possible outcome in a measurement of $X$ ) is given by

$$
p_{n}(X)=\operatorname{Tr}\left(P_{n}(X) \rho\right)
$$

where $P_{n}(X)$ denotes the projection operator on the $n$th eigenspace of $X$.

The uncertainty principle states that, for general pairs of observables $X$ and $Y$, the outcomes of a simultaneous measurement cannot both be fixed with arbitrary precision. One 
way to express this fact mathematically is through the Landau-Pollak uncertainty relation,

$$
\arccos \sqrt{\max _{n} p_{n}(X)}+\arccos \sqrt{\max _{n} p_{n}(Y)} \geq \arccos c,
$$

where

$$
c=c(X, Y) \equiv \max _{i, j}\left|\left\langle x_{i} \mid y_{j}\right\rangle\right|
$$

The relevance of this inequality in quantum mechanics was first pointed out by Uffink [14], who translated to the quantum language the original work of Landau and Pollak on uncertainty in signal theory [15].

The expressions

$$
M_{r}(\mathcal{P})=\left(\sum_{n=1}^{N}\left(p_{n}\right)^{1+r}\right)^{1 / r}, \quad r>-1,
$$

measure the concentration of the probability distribution $\mathcal{P}=\left(p_{1}, p_{2}, \ldots, p_{N}\right)$. They are closely related to the Rényi entropies [16],

$$
H_{q}^{(R)}(\mathcal{P})=\frac{1}{1-q} \ln \left(\sum_{n=1}^{N}\left(p_{n}\right)^{q}\right), \quad q>0,
$$

and the Tsallis entropies [17],

$$
H_{q}^{(T)}(\mathcal{P})=\frac{1}{1-q}\left(\sum_{n=1}^{N}\left(p_{n}\right)^{q}-1\right), \quad q>0,
$$

both of which include the usual (Shannon) entropy as the particular case $q=1$. The quantities $M_{r}(\mathcal{P})$ were first used as measures of uncertainty in quantum mechanics in Refs. 12, 14], where a summary of their properties is given; a more detailed analysis can be found in [18]. Here we will just mention that $M_{r}(\mathcal{P})$ is a continuous non-decreasing function of $r$, with the limiting value

$$
M_{\infty}(\mathcal{P})=\max _{n} p_{n}
$$

and $M_{r}(\mathcal{P})$ is convex in $\mathcal{P}$, i.e., for $0 \leq \lambda \leq 1$,

$$
M_{r}\left(\lambda \mathcal{P}_{1}+(1-\lambda) \mathcal{P}_{2}\right) \leq \lambda M_{r}\left(\mathcal{P}_{1}\right)+(1-\lambda) M_{r}\left(\mathcal{P}_{2}\right)
$$

Taking into account Eq. (9), the Landau-Pollak uncertainty relation (4) can be written as

$$
\arccos \sqrt{M_{\infty}(X)}+\arccos \sqrt{M_{\infty}(Y)} \geq \arccos c
$$


Maximizing the sum $M_{\infty}(X)+M_{\infty}(Y)$ under the constraint (11), we obtain the uncertainty inequality

$$
M_{\infty}(X)+M_{\infty}(Y) \leq 1+c,
$$

which is weaker than (11) but has a simpler and more natural form.

\section{SEPARABILITY CONDITIONS FOR TWO-QUBIT SYSTEMS}

Consider the following observables acting on a bipartite two-dimensional Hilbert space,

$$
Z=\sigma_{z}^{A} \otimes \sigma_{z}^{B}, \quad X=\sigma_{x}^{A} \otimes \sigma_{x}^{B},
$$

where $\sigma_{i}^{j}(i=x, y, z ; j=A, B)$ are the standard Pauli operators acting on the $j$ qubit. Since $Z$ and $X$ commute, for this pair of observables we have that $c=1$, and the right-hand side of (111) vanishes imposing no restriction on the possible outcomes of measurements. The trivial lower bound 0 in Eq. (111) is attained, for instance, if the measured state is one of the four maximally entangled elements of the Bell basis,

$$
\begin{aligned}
\left|\phi^{ \pm}\right\rangle & =\frac{1}{\sqrt{2}}(|00\rangle \pm|11\rangle), \\
\left|\psi^{ \pm}\right\rangle & =\frac{1}{\sqrt{2}}(|01\rangle \pm|10\rangle),
\end{aligned}
$$

where we consider $|0\rangle$ and $|1\rangle$ to be eigenvectors of $\sigma_{z}$ corresponding to the eigenvalues +1 and -1 , respectively.

However, if $Z$ and $X$ act on a separable state, the lower bound 0 is not attainable, which enables the possibility of obtaining a separability condition. This can be done by using Lemma 1 of [10], which we quote here:

Let $\rho=\rho_{A} \otimes \rho_{B}$ be a product state on a bipartite Hilbert space $H=H_{A} \otimes H_{B}$, and let $A(B)$ be observables with nonzero eigenvalues on $H_{A}\left(H_{B}\right)$. Then

$$
\begin{aligned}
& \mathcal{P}(A \otimes B, \rho) \prec \mathcal{P}\left(A, \rho_{A}\right), \\
& \mathcal{P}(A \otimes B, \rho) \prec \mathcal{P}\left(B, \rho_{B}\right)
\end{aligned}
$$

holds. The notation $\mathcal{P} \succ \mathcal{Q}$ ("P $\mathcal{P}$ majorizes $\mathcal{Q}$ ") means that, if $\mathcal{P}=\left(p_{1}, p_{2}, \ldots, p_{N}\right)$ and $\mathcal{Q}=\left(q_{1}, q_{2}, \ldots, q_{N}\right)$ denote two probability distributions written in decreasing order (i.e. $p_{1} \geq p_{2} \geq \ldots \geq p_{N}$ and $\left.q_{1} \geq q_{2} \geq \ldots \geq q_{N}\right)$, then

$$
\sum_{i=1}^{k} p_{i} \geq \sum_{i=1}^{k} q_{i}
$$


for all $k \in[1, \ldots, N]$.

It follows from the previous definition that Eq. (15) implies the inequalities

$$
\begin{aligned}
& M_{\infty}(A \otimes B, \rho) \leq M_{\infty}\left(A, \rho_{A}\right), \\
& M_{\infty}(A \otimes B, \rho) \leq M_{\infty}\left(B, \rho_{B}\right) .
\end{aligned}
$$

Therefore, if $\rho_{\text {sep }}$ denotes an arbitrary (mixed) separable state, i.e. $\rho_{\text {sep }}=\sum_{i} p_{i} \rho_{i}^{A} \otimes \rho_{i}^{B}$, and $A_{1}, A_{2}, B_{1}, B_{2}$ are observables with nonzero eigenvalues, we have that

$$
\begin{aligned}
& M_{\infty}\left(A_{1} \otimes B_{1}, \rho_{\text {sep }}\right)+M_{\infty}\left(A_{2} \otimes B_{2}, \rho_{\text {sep }}\right) \\
& \leq \sum_{i} p_{i}\left(M_{\infty}\left(A_{1} \otimes B_{1}, \rho_{i}^{A} \otimes \rho_{i}^{B}\right)+M_{\infty}\left(A_{2} \otimes B_{2}, \rho_{i}^{A} \otimes \rho_{i}^{B}\right)\right) \\
& \leq \sum_{i} p_{i}\left(M_{\infty}\left(A_{1}, \rho_{i}^{A}\right)+M_{\infty}\left(A_{2}, \rho_{i}^{A}\right)\right) \\
& \leq \sum_{i} p_{i}\left(1+c\left(A_{1}, A_{2}\right)\right)=1+c\left(A_{1}, A_{2}\right),
\end{aligned}
$$

where we have used Eqs. (10) and (12) in addition to (17). Since both $\sigma_{z}$ and $\sigma_{x}$ have the eigenvalues +1 and -1 , they satisfy the conditions of the above lemma, and use of Eq. (18) with the well-known value $c\left(\sigma_{z}, \sigma_{x}\right)=1 / \sqrt{2}$ gives

$$
M_{\infty}\left(Z, \rho_{\text {sep }}\right)+M_{\infty}\left(X, \rho_{\text {sep }}\right) \leq 1+\frac{1}{\sqrt{2}} \approx 1.71
$$

We have seen that the method developed by Gühne and Lewenstein in 10] to derive separability conditions from entropic uncertainty relations can also be applied to the LandauPollak uncertainty relation. However, as we shall prove in the following, inequality (19) can be improved by performing a direct maximization of the sum of $M_{\infty}(Z)$ and $M_{\infty}(X)$ in product states; the bound attained in this way will be valid for any separable state because of the convexity of $M_{\infty}$.

An arbitrary product state is of the form (11) with

$$
\begin{gathered}
|\phi\rangle_{A}=\cos \alpha|0\rangle_{A}+e^{i \delta} \sin \alpha|1\rangle_{A}, \\
|\varphi\rangle_{B}=\cos \beta|0\rangle_{B}+e^{i \gamma} \sin \beta|1\rangle_{B},
\end{gathered}
$$

where $\alpha, \beta \in[0, \pi / 2]$ and $\delta, \gamma \in[0,2 \pi)$. Both $Z$ and $X$ have the eigenvalues +1 and -1 , 
and the corresponding eigenspace projectors are

$$
\begin{aligned}
& P_{+}(Z)=|00\rangle\langle 00|+| 11\rangle\langle 11|, \\
& P_{-}(Z)=|01\rangle\langle 01|+| 10\rangle\langle 10|, \\
& P_{ \pm}(X)=\left|\phi^{ \pm}\right\rangle\left\langle\phi^{ \pm}|+| \psi^{ \pm}\right\rangle\left\langle\psi^{ \pm}\right| .
\end{aligned}
$$

Therefore, according to Eq. (3), the probabilities of finding the pure separable state (1201) in these eigenspaces are, respectively,

$$
\begin{aligned}
& p_{+}(Z)=(\cos \alpha \cos \beta)^{2}+(\sin \alpha \sin \beta)^{2}, \\
& p_{-}(Z)=1-(\cos \alpha \cos \beta)^{2}-(\sin \alpha \sin \beta)^{2}, \\
& p_{ \pm}(X)=\frac{1}{2}(1 \pm \cos \delta \cos \gamma \sin 2 \alpha \sin 2 \beta) .
\end{aligned}
$$

Since $p_{ \pm}(Z)$ do not depend on $\delta$ and $\gamma$, and $\sin 2 \alpha \sin 2 \beta$ is always nonnegative, the maximum value of $M_{\infty}(Z)+M_{\infty}(X)$ equals the maximum of the functions

$$
f_{ \pm}(\alpha, \beta)=p_{ \pm}(Z)+\frac{1}{2}(1+\sin 2 \alpha \sin 2 \beta)
$$

which occurs when $\alpha= \pm \beta$. Thus we find our first necessary separability condition,

$$
M_{\infty}\left(Z, \rho_{s e p}\right)+M_{\infty}\left(X, \rho_{s e p}\right) \leq \frac{3}{2}
$$

If for a certain state $M_{\infty}(Z)+M_{\infty}(X)>3 / 2$, then Eq. (24) implies that the state is entangled.

As shown in [9], the introduction of a third observable,

$$
Y=\sigma_{y}^{A} \otimes \sigma_{y}^{B}
$$

enables the possibility of obtaining a more accurate separability condition, due to the fact that we are then using the maximal number of complementary observables available for each subsystem [19]. Unfortunately, no generalization of the Landau-Pollak uncertainty relation is known for sets of more than two observables (leaving aside the one that is trivially obtained from Eq. (12)), which prevents us from using Gühne and Lewenstein's method in this case. Therefore, we will follow the direct maximization procedure in order to set an upper bound for the sum of $M_{\infty}(X), M_{\infty}(Y)$, and $M_{\infty}(Z)$ in separable states.

Observable $Y$ has the same eigenvalues as $Z$ and $X$, with eigenspace projectors

$$
P_{ \pm}(Y)=\left|\phi^{\mp}\right\rangle\left\langle\phi^{\mp}|+| \psi^{ \pm}\right\rangle\left\langle\psi^{ \pm}\right|
$$


and the corresponding probabilities for the pure separable state (120) are

$$
p_{ \pm}(Y)=\frac{1}{2}(1 \pm \sin \delta \sin \gamma \sin 2 \alpha \sin 2 \beta) .
$$

Since $\sin 2 \alpha \sin 2 \beta$ is nonnegative, and the maximum over $\delta$ and $\gamma$ of the four functions of the form $\pm(\sin \delta \sin \gamma \pm \cos \delta \cos \gamma)= \pm \cos (\delta \mp \gamma)$ equals 1 , we only have to find the maximum of the functions

$$
\begin{aligned}
g_{ \pm}(\alpha, \beta) & =p_{ \pm}(Z)+1+\frac{\sin 2 \alpha \sin 2 \beta}{2} \\
& =f_{ \pm}(\alpha, \beta)+\frac{1}{2} .
\end{aligned}
$$

Recalling the derivation of Eq. (24), we obtain our second necessary separability condition,

$$
M_{\infty}\left(X, \rho_{\text {sep }}\right)+M_{\infty}\left(Y, \rho_{\text {sep }}\right)+M_{\infty}\left(Z, \rho_{\text {sep }}\right) \leq 2
$$

Taking into account that $M_{\infty}\left(Y, \rho_{\text {sep }}\right) \geq 1 / 2$, we see that condition (24) can be derived from (29), so that the latter is stronger than the former.

Attending to [7, 9], the best separability conditions are obtained by choosing as observables the three orthogonal components of the total spin of the system,

$$
S_{i}=\sigma_{i}^{A} \otimes \mathbb{I}_{B}+\mathbb{I}_{A} \otimes \sigma_{i}^{B} \quad(i=x, y, z),
$$

where II denotes the identity operator. These observables all have the eigenvalues \pm 2 (nondegenerate) and 0 (two-time degenerate), with eigenspace projectors

$$
\begin{aligned}
& P_{ \pm}\left(S_{x}\right)=\frac{1}{2}\left(\left|\phi^{+}\right\rangle \pm\left|\psi^{+}\right\rangle\right)\left(\left\langle\phi^{+}\right| \pm\left\langle\psi^{+}\right|\right), \\
& P_{0}\left(S_{x}\right)=\left|\phi^{-}\right\rangle\left\langle\phi^{-}|+| \psi^{-}\right\rangle\left\langle\psi^{-}\right|, \\
& P_{ \pm}\left(S_{y}\right)=\frac{1}{2}\left(\left|\phi^{-}\right\rangle \pm\left|\psi^{+}\right\rangle\right)\left(\left\langle\phi^{-}\right| \pm\left\langle\psi^{+}\right|\right), \\
& P_{0}\left(S_{y}\right)=\left|\phi^{+}\right\rangle\left\langle\phi^{+}|+| \psi^{-}\right\rangle\left\langle\psi^{-}\right|, \\
& P_{+}\left(S_{z}\right)=|00\rangle\left\langle 00\left|, \quad P_{-}\left(S_{z}\right)=\right| 11\right\rangle\langle 11|, \\
& P_{0}\left(S_{z}\right)=|01\rangle\langle 01|+| 10\rangle\langle 10|,
\end{aligned}
$$


and the corresponding probabilities for the generic pure state (120) are

$$
\begin{aligned}
& p_{ \pm}\left(S_{x}\right)=\frac{1}{4}(1 \pm \cos \delta \sin 2 \alpha)(1 \pm \cos \gamma \sin 2 \beta), \\
& p_{0}\left(S_{x}\right)=\frac{1}{2}(1-\cos \delta \cos \gamma \sin 2 \alpha \sin 2 \beta), \\
& p_{ \pm}\left(S_{y}\right)=\frac{1}{4}(1 \pm \sin \delta \sin 2 \alpha)(1 \pm \sin \gamma \sin 2 \beta), \\
& p_{0}\left(S_{y}\right)=\frac{1}{2}(1-\sin \delta \sin \gamma \sin 2 \alpha \sin 2 \beta), \\
& p_{+}\left(S_{z}\right)=(\cos \alpha \cos \beta)^{2}, \quad p_{-}\left(S_{z}\right)=(\sin \alpha \sin \beta)^{2}, \\
& p_{0}\left(S_{z}\right)=(\cos \alpha \sin \beta)^{2}+(\sin \alpha \cos \beta)^{2} .
\end{aligned}
$$

We therefrom see that the maximum value of $\sum_{i} M_{\infty}\left(S_{i}\right)$ for product states is the maximum of

$$
w(\alpha, \beta)=p\left(S_{z}\right)+1+\frac{\sin 2 \alpha \sin 2 \beta}{2},
$$

which is easily found to be equal to 2 . Thus we get our third necessary separability condition,

$$
M_{\infty}\left(S_{x}, \rho_{\text {sep }}\right)+M_{\infty}\left(S_{y}, \rho_{\text {sep }}\right)+M_{\infty}\left(S_{z}, \rho_{\text {sep }}\right) \leq 2
$$

Another interesting possibility is that of measuring a non-degenerate Bell diagonal observable,

$$
B=\lambda_{1}\left|\phi^{+}\right\rangle\left\langle\phi^{+}\left|+\lambda_{2}\right| \phi^{-}\right\rangle\left\langle\phi^{-}\left|+\lambda_{3}\right| \psi^{+}\right\rangle\left\langle\psi^{+}\left|+\lambda_{4}\right| \psi^{-}\right\rangle\left\langle\psi^{-}\right|,
$$

with $\lambda_{i} \neq \lambda_{j}$ when $i \neq j$. The probability distribution for the outcomes of $B$ acting on the pure separable state (120) is

$$
\begin{aligned}
& p_{\phi^{ \pm}}(B)=\frac{1}{2}\left[(\cos \alpha \cos \beta)^{2}+(\sin \alpha \sin \beta)^{2} \pm \xi(\alpha, \beta) \zeta_{+}(\delta, \gamma)\right], \\
& p_{\psi^{ \pm}}(B)=\frac{1}{2}\left[(\cos \alpha \sin \beta)^{2}+(\sin \alpha \cos \beta)^{2} \pm \xi(\alpha, \beta) \zeta_{-}(\delta, \gamma)\right],
\end{aligned}
$$

where $\xi(\alpha, \beta)=\frac{1}{2} \sin 2 \alpha \sin 2 \beta$ and $\zeta_{ \pm}(\delta, \gamma)=\cos (\delta \pm \gamma)$. The nonnegativity of $\xi(\alpha, \beta)$ implies that $M_{\infty}(B)$ is the maximum over $\alpha$ and $\beta$ of the functions

$$
\begin{aligned}
& h_{1}(\alpha, \beta)=\frac{1}{2}\left[(\cos \alpha \cos \beta)^{2}+(\sin \alpha \sin \beta)^{2}+\xi(\alpha, \beta)\right], \\
& h_{2}(\alpha, \beta)=\frac{1}{2}\left[(\cos \alpha \sin \beta)^{2}+(\sin \alpha \cos \beta)^{2}+\xi(\alpha, \beta)\right],
\end{aligned}
$$

and, therefore,

$$
M_{\infty}(B) \leq \frac{1}{2} .
$$


This last necessary separability condition is not new, since it was previously derived by Gühne and Lewenstein [10] using a different method. As pointed out by these authors, condition (38) is equivalent to the set of four optimal EW's

$$
W_{\phi^{ \pm}}=\frac{1}{2} \mathbb{I}-\left|\phi^{ \pm}\right\rangle\left\langle\phi^{ \pm}\left|, \quad W_{\psi^{ \pm}}=\frac{1}{2} \mathbb{I}-\right| \psi^{ \pm}\right\rangle\left\langle\psi^{ \pm}\right|
$$

\section{ACCURACY OF THE SEPARABILITY CONDITIONS}

Next we will test the power as entanglement detectors of the separability conditions derived in the previous section, by applying them to states whose separability limits are already known. We will also compare our separability conditions with previous criteria. All the probabilities below are calculated using Eq. (3) and the projectors found in Sec. III.

\section{A. Werner states}

Werner states [20] are mixtures of a completely random state and a maximally entangled pure state. In the case of two qubits, and choosing the maximally entangled state to be the singlet state, they read

$$
\rho_{W}=\frac{1-p}{4} \mathbb{I}_{A} \otimes \mathbb{I}_{B}+p\left|\psi^{-}\right\rangle\left\langle\psi^{-}\right|
$$

where $p \in[0,1]$. These states are known to be separable iff $p \leq 1 / 3$ (see [21] and references therein). The probabilities of finding $\rho_{W}$ in each eigenspace when measuring the observables of Sec. III are

$$
\begin{aligned}
& p_{ \pm}(X)=p_{ \pm}(Y)=p_{ \pm}(Z)=\frac{1 \mp p}{2} \\
& p_{0}\left(S_{i}\right)=\frac{1+p}{2} \\
& p_{ \pm}\left(S_{i}\right)=p_{\phi^{ \pm}}(B)=p_{\psi^{+}}(B)=\frac{1-p}{4} \\
& p_{\psi^{-}}(B)=\frac{1+3 p}{4}
\end{aligned}
$$


Thus we have,

$$
\begin{aligned}
& \sum_{\tau=X, Z} M_{\infty}\left(\tau, \rho_{W}\right)=1+p \\
& \sum_{\tau=X, Y, Z} M_{\infty}\left(\tau, \rho_{W}\right)=\frac{3(1+p)}{2}, \\
& \sum_{i=x, y, z} M_{\infty}\left(S_{i}, \rho_{W}\right)=\frac{3(1+p)}{2} \\
& M_{\infty}\left(B, \rho_{W}\right)=\frac{1+3 p}{4}
\end{aligned}
$$

We see from these results that the separability condition (24) detects entanglement when $p>1 / 2$, while (29), (34), and (38) detect entanglement when $p>1 / 3$. It is worth noting that in this case the three latter separability conditions, like variance-based criteria [7], are optimal in the sense that they are able to detect all the entangled states. All four conditions improve the bound obtained in [9] using Shannon entropies $(p>0.55)$, as well as those derived in [10] by means of Tsallis entropies $(p>1 / \sqrt{3})$ and Bell's inequality criterion $(p>1 / \sqrt{2})$. Even more, when measuring the same observables (i.e. when using the same experimental setting), our conditions always improve on the bounds given by the Shannon and Tsallis entropic conditions, respectively: $p>0.78$ and $p>1 / \sqrt{2}$ when measuring $X$ and $Z ; p>0.65$ and $p>1 / \sqrt{3}$ when measuring $X, Y$, and $Z ; p>0.55$ when measuring $S_{x}$, $S_{y}$, and $S_{z}$; and $p>0.74$ when measuring $B$ (in the last two cases only Shannon entropic conditions are available).

\section{B. Gisin states}

Gisin states [22] are mixtures of the same fraction of the pure states $|00\rangle$ and $|11\rangle$, and any pure superposition of the states $|01\rangle$ and $|10\rangle$. That is,

$$
\rho_{G}=p|\chi\rangle\langle\chi|+\frac{1-p}{2}(|00\rangle\langle 00|+| 11\rangle\langle 11|)
$$

where $|\chi\rangle=\cos \alpha|01\rangle+e^{i \beta} \sin \alpha|10\rangle, \alpha \in[0, \pi / 2], \beta \in[0,2 \pi)$, and $p \in[0,1]$. The state $\rho_{G}$ is known to be separable iff [3]

$$
p \leq \frac{1}{1+\sin 2 \alpha}
$$


In this case,

$$
\begin{aligned}
& p_{ \pm}(X)=p_{ \pm}(Y)=\frac{1 \pm p \sin 2 \alpha \cos \beta}{2}, \\
& p_{+}(Z)=1-p, \quad p_{-}(Z)=p, \\
& p_{ \pm}\left(S_{x}\right)=p_{ \pm}\left(S_{y}\right)=\frac{1}{2} p_{+}(X), \\
& p_{0}\left(S_{x}\right)=p_{0}\left(S_{y}\right)=p_{-}(X), \\
& p_{ \pm}\left(S_{z}\right)=p_{\phi^{ \pm}}(B)=\frac{1-p}{2}, \quad p_{0}\left(S_{z}\right)=p, \\
& p_{\psi^{ \pm}}(B)=\frac{p(1 \pm \sin 2 \alpha \cos \beta)}{2},
\end{aligned}
$$

which leads to

$$
\begin{aligned}
& \sum_{\tau=X, Z} M_{\infty}\left(\tau, \rho_{G}\right)=\max \{p, 1-p\}+\frac{1+p \sin 2 \alpha|\cos \beta|}{2} \\
& \sum_{\tau=X, Y, Z} M_{\infty}\left(\tau, \rho_{G}\right)=\max \{p, 1-p\}+1+p \sin 2 \alpha|\cos \beta| \\
& \sum_{i=x, y, z} M_{\infty}\left(S_{i}, \rho_{G}\right)=\max \left\{p, \frac{1-p}{2}\right\} \\
& +2 \max \left\{\frac{1-p \sin 2 \alpha \cos \beta}{2}, \frac{1+p \sin 2 \alpha \cos \beta}{4}\right\} \\
& M_{\infty}(B)=\max \left\{\frac{1-p}{2}, \frac{p(1+\sin 2 \alpha|\cos \beta|)}{2}\right\}
\end{aligned}
$$

These results imply that conditions (24), (29), (34), and (38) detect entanglement when $p>\left(1+\frac{1}{2} \sin 2 \alpha|\cos \beta|\right)^{-1}, p>(1+\sin 2 \alpha|\cos \beta|)^{-1}, p>(1-\sin 2 \alpha \cos \beta)^{-1}$, and $p>$ $(1+\sin 2 \alpha|\cos \beta|)^{-1}$, respectively (notice that the restriction imposed by (34) is meaningful only when $\beta \in(\pi / 2,3 \pi / 2))$. Thus we find that in this case the best separability conditions are (29) and (38), though in general they are not optimal. When $\beta=0, \pi$ all entangled states are detected by (29) and (38), but as $\beta$ departs from these values the separability conditions fail to detect an increasing amount of entangled states, until for $\beta=\pi / 2,3 \pi / 2$ no entanglement is detected. For values of $\beta$ such that $|\cos \beta|>\sqrt{2}-1$, conditions (29) and (38) improve the bound given by Bell's inequality criterion, $p>(1+(\sqrt{2}-1) \sin 2 \alpha)^{-1}$ [22]. It is worth noting that, due to the dependence of the probabilities on two parameters, to establish which states are detected by the entropic separability criteria is mathematically cumbersome, and has to be carried out by numerical analysis. 


\section{Mixtures of a singlet and a maximally polarized pair}

The states

$$
\rho_{0}=p\left|\psi^{-}\right\rangle\left\langle\psi^{-}|+(1-p)| 00\right\rangle\langle 00|,
$$

with $p \in[0,1]$, are known by the positive partial transpose criterion to be separable only if $p=0$ [3]. The probabilities for the observables of Sec. III are now

$$
\begin{aligned}
& p_{ \pm}(X)=p_{ \pm}(Y)=\frac{1 \mp p}{2}, \\
& p_{0}\left(S_{x}\right)=p_{0}\left(S_{y}\right)=\frac{1+p}{2}, \\
& p_{ \pm}\left(S_{x}\right)=p_{ \pm}\left(S_{y}\right)=\frac{1-p}{4}, \\
& p_{+}(Z)=p_{+}\left(S_{z}\right)=1-p, \\
& p_{-}(Z)=p_{0}\left(S_{z}\right)=p_{\psi^{-}}(B)=p, \\
& p_{-}\left(S_{z}\right)=p_{\psi^{+}}(B)=0, \\
& p_{\phi^{ \pm}}(B)=\frac{1-p}{2},
\end{aligned}
$$

and, therefore,

$$
\begin{aligned}
& \sum_{\tau=X, Z} M_{\infty}\left(\tau, \rho_{0}\right)=\max \{p, 1-p\}+\frac{1+p}{2}, \\
& \sum_{\tau=X, Y, Z} M_{\infty}\left(\tau, \rho_{0}\right)=\max \{p, 1-p\}+1+p, \\
& \sum_{i=x, y, z} M_{\infty}\left(S_{i}, \rho_{0}\right)=\max \{p, 1-p\}+1+p, \\
& M_{\infty}(B)=\max \left\{p, \frac{1-p}{2}\right\} .
\end{aligned}
$$

We thus find that condition (24) detects entanglement for $p>2 / 3$, while it suffices to have $p>1 / 2$ in order to detect entangled states using conditions (29), (34), and (38). These bounds are not optimal, but they improve on that derived from the violation of Bell's inequality, $p>0.8[3]$. Furthermore, as in the case of Werner states, in each measurement setting the bounds provided by our conditions are better than those obtained using Shannon entropies: $p>0.85$ when measuring $X$ and $Z ; p>0.73$ when measuring $X, Y$, and $Z$; $p>0.55$ when measuring $S_{x}, S_{y}$, and $S_{z}$; and $p>0.78$ when measuring $B$. 


\section{EQUIVALENCE OF CONDITION (29) AND THE SET OF OPTIMAL EW'S} (39)

The necessary separability condition (38) is equivalent to the set of optimal EW's (39) [10]. Using the three-dimensional space representation of density matrices with coordinates $\operatorname{Tr}(X \rho), \operatorname{Tr}(Y \rho)$, and $\operatorname{Tr}(Z \rho)$ (see Refs. [10] and [23]), this equivalence means that condition (38) is able to recognize the octahedron containing all separable states, which lies inside the tetrahedron whose vertices are the Bell states and contains all possible states.

For the three families of states considered in the previous section, the separability conditions (29) and (38) detect the same entangled states, which suggests that they are equivalent. In the following we will prove that this is indeed the case, so that condition (29) is also equivalent to the set of optimal EW's (39) and has the same success at detecting the octahedron that contains the separable states.

Condition (38) can be stated as

$$
0 \leq \operatorname{Tr}\left(\left|B S_{i}\right\rangle\left\langle B S_{i}\right| \rho_{\text {sep }}\right) \leq \frac{1}{2}
$$

where $\left|B S_{i}\right\rangle$ is any element of the Bell basis (14). Taking into account the identities [10]

$$
\begin{aligned}
& \operatorname{Tr}\left(\left|\phi^{ \pm}\right\rangle\left\langle\phi^{ \pm}\right| \rho\right)=\frac{1 \pm \operatorname{Tr}(X \rho) \mp \operatorname{Tr}(Y \rho)+\operatorname{Tr}(Z \rho)}{4}, \\
& \operatorname{Tr}\left(\left|\psi^{ \pm}\right\rangle\left\langle\psi^{ \pm}\right| \rho\right)=\frac{1 \pm \operatorname{Tr}(X \rho) \pm \operatorname{Tr}(Y \rho)-\operatorname{Tr}(Z \rho)}{4}
\end{aligned}
$$

and noting that

$$
\operatorname{Tr}(\tau \rho)=p_{+}(\tau)-p_{-}(\tau) \equiv \Delta p(\tau) \quad(\tau=X, Y, Z)
$$

the inequalities in (50) can be written as

$$
\begin{aligned}
& -1 \leq \pm \Delta p\left(X, \rho_{\text {sep }}\right) \mp \Delta p\left(Y, \rho_{\text {sep }}\right)+\Delta p\left(Z, \rho_{\text {sep }}\right) \leq 1, \\
& -1 \leq \pm \Delta p\left(X, \rho_{\text {sep }}\right) \pm \Delta p\left(Y, \rho_{\text {sep }}\right)-\Delta p\left(Z, \rho_{\text {sep }}\right) \leq 1 .
\end{aligned}
$$

This is equivalent to the eight inequalities of the form

$$
-1 \leq \pm \Delta p\left(X, \rho_{\text {sep }}\right) \pm \Delta p\left(Y, \rho_{\text {sep }}\right) \pm \Delta p\left(Z, \rho_{\text {sep }}\right) \leq 1
$$

that is,

$$
\left|\Delta p\left(X, \rho_{\text {sep }}\right)\right|+\left|\Delta p\left(Y, \rho_{\text {sep }}\right)\right|+\left|\Delta p\left(Z, \rho_{\text {sep }}\right)\right| \leq 1 .
$$


Finally, noting that for $\tau=X, Y, Z$

$$
|\Delta p(\tau)|=M_{\infty}(\tau)-\left(1-M_{\infty}(\tau)\right)=2 M_{\infty}(\tau)-1
$$

Eq. (55) reduces to (29), which proves that this separability condition is equivalent to (38).

\section{SEPARABILITY CONDITIONS FOR MORE COMPLEX SYSTEMS}

If we consider multipartite and/or higher-dimensional systems (qudits), the direct maximization procedure used in Sec. III for two-qubit systems becomes too complicated to be carried out analytically, due to the increasing number of free parameters, although it can be faced numerically. However, the method of Gühne and Lewenstein (see Sec. III) can also be applied in this case, and allows us to derive separability conditions from the Landau-Pollak uncertainty relation.

\section{A. Bipartite systems of qudits}

For states of a two-dimensional Hilbert space, the best detection of entanglement is achieved by measuring in each subsystem the three orthogonal components of spin, which are also a maximal set of complementary observables. We recall that two observables $A, B$

in $D$-dimensional Hilbert space are said to be complementary if $c(A, B)=1 / \sqrt{D}[24]$, and maximal sets of $D+1$ pairwise complementary observables are known to exist when $D$ is either a prime [25] or a power of a prime [19]. However, when the dimension of the Hilbert space is greater than two, the orthogonal components of spin are not complementary observables and both cases must be treated separately.

Choosing $A_{1}, A_{2}$ and/or $B_{1}, B_{2}$ to be complementary observables in $D$-dimensional Hilbert space, we find from Eq. (18) that

$$
M_{\infty}\left(A_{1} \otimes B_{1}, \rho_{\text {sep }}\right)+M_{\infty}\left(A_{2} \otimes B_{2}, \rho_{\text {sep }}\right) \leq 1+\frac{1}{\sqrt{D}}
$$

On the other hand, if $S_{n}$ and $S_{n^{\prime}}$ denote $D$-dimensional spin observables along the axes 
$n$ and $n^{\prime}$, respectively, we have that [26]

$$
\begin{aligned}
& c^{2}\left(S_{n}, S_{n^{\prime}}\right)=\left(\begin{array}{c}
D-1 \\
n^{*}
\end{array}\right)\left(\cos ^{2} \frac{\beta}{2}\right)^{D-1-n^{*}}\left(\sin ^{2} \frac{\beta}{2}\right)^{n^{*}}, \\
& n^{*}=\left[D \sin ^{2} \frac{\beta}{2}\right]
\end{aligned}
$$

where $\beta$ is the angle between the axes $n$ and $n^{\prime}$, and the square brackets denote integer part of the expression within. Therefore, use of Eq. (18) leads to

$$
\begin{aligned}
& M_{\infty}\left(S_{n}^{A} \otimes S_{n}^{B}, \rho_{\text {sep }}\right)+M_{\infty}\left(S_{n^{\prime}}^{A} \otimes S_{n^{\prime}}^{B}, \rho_{\text {sep }}\right) \\
& \leq 1+\sqrt{\left(\begin{array}{c}
D-1 \\
n^{*}
\end{array}\right)}\left(\cos \frac{\beta}{2}\right)^{D-1-n^{*}}\left(\sin \frac{\beta}{2}\right)^{n^{*}},
\end{aligned}
$$

and choosing the axes $n, n^{\prime}$ to be orthogonal $(\beta=\pi / 2)$ the previous inequality simplifies to

$$
\begin{aligned}
& M_{\infty}\left(S_{x}^{A} \otimes S_{x}^{B}, \rho_{\text {sep }}\right)+M_{\infty}\left(S_{z}^{A} \otimes S_{z}^{B}, \rho_{\text {sep }}\right) \\
& \leq 1+\sqrt{\frac{1}{2^{D-1}}\left(\begin{array}{c}
D-1 \\
{[D / 2]}
\end{array}\right)} .
\end{aligned}
$$

It is worth noting that when $D$ is odd the spin observables have one non-degenerate zero eigenvalue, so that the conditions in Gühne and Lewenstein's lemma are not fulfilled. However, as pointed out by these authors [10], the requirement that the observables have nonzero eigenvalues is more a technical condition and can always be achieved by altering the eigenvalues, since the Landau-Pollak uncertainty relation, like the entropic uncertainty relations considered in [10], does not depend on them.

\section{B. Multipartite systems}

In the case of tripartite systems we must distinguish between fully separable states, which are states (or mixtures of states) of the form

$$
|\psi\rangle_{A B C}=|\phi\rangle_{A} \otimes|\varphi\rangle_{B} \otimes|\chi\rangle_{C}
$$

and biseparable states, which are product states with respect to one particular bipartite splitting of the system, e.g.

$$
|\psi\rangle_{A B C}=|\phi\rangle_{A} \otimes|\varphi\rangle_{B C},
$$


or mixtures of states of this form. Fully separable and biseparable states, as well as other kinds of partially separable states, can be defined likewise for general multipartite systems.

A straightforward generalization of Eq. (18) enables us to derive biseparability conditions for multipartite qubit and qudit systems. Thus, for instance, on the analogy of (19) we find the following biseparability condition for systems of three qubits:

$$
\begin{aligned}
& M_{\infty}\left(\sigma_{x}^{A} \otimes \sigma_{x}^{B} \otimes \sigma_{x}^{C}\right)+M_{\infty}\left(\sigma_{z}^{A} \otimes \sigma_{z}^{B} \otimes \sigma_{z}^{C}\right) \\
& \leq 1+\frac{1}{\sqrt{2}} \approx 1.71 .
\end{aligned}
$$

Likewise, the multipartite analogues of Eqs. (57) and (60) are, respectively, the following biseparability conditions for systems with an arbitrary number of subsystems in $D$ dimensional Hilbert space:

$$
\begin{aligned}
& M_{\infty}\left(C_{1}^{A_{1}} \otimes \cdots \otimes C_{1}^{A_{D}}, \rho_{\text {sep }}\right)+M_{\infty}\left(C_{2}^{A_{1}} \otimes \cdots \otimes C_{2}^{A_{D}}, \rho_{\text {sep }}\right) \\
& \leq 1+\frac{1}{\sqrt{D}}
\end{aligned}
$$

where $C_{1}$ and $C_{2}$ are complementary observables, and

$$
\begin{aligned}
& M_{\infty}\left(S_{x}^{A_{1}} \otimes \cdots \otimes S_{x}^{A_{D}}, \rho_{\text {sep }}\right) \\
& \leq 1+\sqrt{\frac{1}{2^{D-1}}\left(\begin{array}{c}
D-1 \\
{[D / 2]}
\end{array}\right)}
\end{aligned}
$$

We emphasize that, as already noted in Sec. III in relation to the two-qubit case, the separability and biseparability conditions obtained in this section cannot be improved by considering measurements of additional observables, due to the fact that no nontrivial generalization of the Landau-Pollak uncertainty relation is known for sets of more than two observables [27].

\section{CONCLUSIONS}

We have derived several necessary separability conditions for two-qubit systems, namely Eqs. (24), (29), (34), and (38), on the basis of the so-called Landau-Pollak uncertainty

relation. Like entropy-based separability criteria, our conditions are expressed in terms of the probability distributions for the outcomes of measurements, so that they can be applied 
in many experimental settings. On the other hand, the measure of uncertainty used here, $M_{\infty}$, is mathematically easier to handle than entropies.

In order to test the power of these conditions as entanglement detectors, we have applied them to three well-known families of two-qubit states, namely Werner states, Gisin states, and mixtures of a singlet and a maximally polarized pair. In most cases, the results obtained are better than those provided by other separability criteria, such as Bell's inequalities violation and entropy-based criteria. Conditions (29), (34), and (38) are even able to detect all entangled two-qubit Werner states, thus improving on entropy-based criteria [9, 10] and

reproducing the results of variance-based criteria [7]. However, the other two families show that in general our conditions are not optimal, i.e. they are necessary but not sufficient. It would be interesting to know whether a refined choice of operators can give optimal results for these states, and, more generally, whether given an entangled state it is always possible to construct a set of observables such that the sum of their $M_{\infty}$ measures is greater in that state than in a generic product state.

We have proved that conditions (29) and (38) are equivalent. Since (38) is known to be equivalent to the set of four optimal EW's (39), the same happens for (29). As a consequence, (29) is able to detect all entangled states lying outside the octahedron of separable states in the three-dimensional representation of density matrices [28]. Condition (24) is weaker than (29), since it does not include the correlations in the third observable; however, we have considered it explicitly because it only needs two measurements and, therefore, it is experimentally less demanding.

Finally, we have extended our results to more complicated cases than two-qubit systems, i.e. to multipartite and higher-dimensional systems, for which no necessary and sufficient condition for entanglement is known to date. The separability conditions obtained in these cases, however, are limited due to the lack of a nontrivial uncertainty relation of LandauPollak type for sets of more than two observables. Therefore, further research in this field might help to improve the results presented here.

\section{Acknowledgments}

The work of the second author (J.S.R.) has been partially supported by Dirección General de Investigación (Ministerio de Ciencia y Tecnología) of Spain under grants BFM2001-3878- 
C02-01 and BFM 2003-06335-C03-02, and the Junta de Andalucía research group FQM-0207.

[1] A. Einstein, N. Podolski, and N. Rosen, Phys. Rev. 47, 777 (1935).

[2] A. K. Ekert, Phys. Rev. Lett. 67, 661 (1991); C. H. Bennett, G. Brassard, C. Crépeau, R. Jozsa, A. Peres, and W. K. Wootters, ibid. 70, 1895 (1993).

[3] A. Peres, Phys. Rev. Lett. 77, 1413 (1996).

[4] A. Peres, Found. Phys. 29, 589 (1999) and references therein.

[5] M. Horodecki, P. Horodecki, and R. Horodecki, Phys. Lett. A 223, 8 (1996); M. Lewenstein, B. Kraus, J. I. Cirac, and P. Horodecki, Phys. Rev. A 62, 052310 (2000).

[6] L.-M. Duan, G. Giedke, J. I. Cirac, and P. Zoller, Phys. Rev. Lett. 84, 2722 (2000).

[7] H. F. Hofmann and S. Takeuchi, Phys. Rev. A 68, 032103 (2003); O. Gühne, Phys. Rev. Lett. 92, 117903 (2004).

[8] H. P. Robertson, Phys. Rev. 34, 163 (1929).

[9] V. Giovannetti, Phys. Rev. A 70, 012102 (2004).

[10] O. Gühne and M. Lewenstein, Phys. Rev. A 70, 022316 (2004).

[11] D. Deutsch, Phys. Rev. Lett. 50, 631 (1983).

[12] H. Maassen and J. B. M. Uffink, Phys. Rev. Lett. 60, 1103 (1988).

[13] J. Sánchez-Ruiz, Phys. Lett. A 173, 233 (1993); J. Phys. A: Math. Gen. 27, L843 (1994); Phys. Lett. A 201, 125 (1995).

[14] J. B. M. Uffink, Ph. D. Thesis, University of Utrecht, 1990.

[15] H. J. Landau and H. O. Pollak, Bell Syst. Tech. J. 40, 65 (1961).

[16] A. Rényi, Probability Theory (North-Holland, Amsterdam, 1970).

[17] C. Tsallis, J. Stat. Phys. 52, 479 (1988).

[18] G. H. Hardy, J. E. Littlewood, and G. Pólya, Inequalities (Cambridge University Press, Cambridge, 1952).

[19] W. K. Wootters and B. D. Fields, Ann. Phys. (N.Y.) 191, 363 (1989).

[20] R. F. Werner, Phys. Rev. A 40, 4277 (1989).

[21] A. O. Pittenger and M. H. Rubin, Opt. Commun. 179, 447 (2000)

[22] N. Gisin, Phys. Lett. A 210, 151 (1996).

[23] R. Horodecki and M. Horodecki, Phys. Rev. A 54, 1838 (1996); R. A. Bertlmann, H. Narnhofer, 
and W. Thirring, ibid. 66, 032319 (2002).

[24] J. Schwinger, Proc. Natl. Acad. Sci. U.S.A. 46, 570 (1960); reprinted in: Quantum Kinematics and Dynamics (Benjamin, New York, 1970).

[25] I. D. Ivanović, J. Phys. A: Math. Gen. 14, 3241 (1981).

[26] J. Sánchez-Ruiz, Phys. Lett. A 181, 193 (1993).

[27] On the contrary, nontrivial entropic uncertainty relations are known for maximal sets of complementary observables in arbitrary dimension [13]. However, in Ref. [10] the authors do not seem to take advantage of this fact for the case of tripartite systems of qubits, since they just consider a three-observable inequality that can be deduced trivially from a two-observable one. It is also worth noting that spin observables might have been used as well to derive separability conditions for qudit systems from entropic uncertainty relations.

[28] Recently, O. Gühne and M. Lewenstein have pointed out in quant-ph/0409140 that variancebased separability conditions can be viewed as nonlinear EW's which improve the linear optimal EW's. 